\title{
Seasonality patterns of mood and behavior in the Old Order Amish
}

\author{
Falguni Patel, \\ Mood and Anxiety Program, Department of Psychiatry, School of Medicine, University of
} Maryland, Baltimore, MD, USA

\section{Nadine Postolache,}

Mood and Anxiety Program, Department of Psychiatry, School of Medicine, University of Maryland, Baltimore, MD, USA

\section{Hira Mohyuddin,}

Mood and Anxiety Program, Department of Psychiatry, School of Medicine, University of Maryland, Baltimore, MD, USA

\section{Dipika Vaswani, \\ Mood and Anxiety Program, Department of Psychiatry, School of Medicine, University of Maryland, Baltimore, MD, USA}

\section{Theodora Balis,}

Mood and Anxiety Program, Department of Psychiatry, School of Medicine, University of Maryland, Baltimore, MD, USA

\author{
Uttam K. Raheja, and \\ Mood and Anxiety Program, Department of Psychiatry, School of Medicine, University of \\ Maryland, Baltimore, MD, USA; and Saint Elizabeths Hospital, Psychiatry Residency Training \\ Program, Washington, DC, USA \\ Teodor T. Postolache \\ Mood and Anxiety Program, Department of Psychiatry, School of Medicine, University of \\ Maryland, 685 W. Baltimore Street, MSTF Room 930, Baltimore, MD 21201, USA
}

\section{Abstract}

\begin{abstract}
Background-Although humans have become partially isolated from physical seasonal environmental changes through artificial lighting and temperature control, seasonal changes in mood and behavior have been described across hemispheres, continents, ethnicities and occupations. The Old Order Amish are more exposed than the general population to environmental seasonal changes both occupationally as well as through their limited use of electric light in the winter and air conditioning in the summer; yet, their seasonal changes in mood and behavior have not been previously studied.
\end{abstract}

Methods-The aim of this study was to analyze seasonal patterns in mood and behavior in the Old Order Amish of Lancaster County, Pennsylvania, who returned completed Seasonal Pattern

\footnotetext{
*Corresponding author: tpostolache@psych.umaryland.edu.

Conflict of interest statement

Authors' conflict of interest disclosure: The authors stated that there are no conflicts of interest regarding the publication of this article.

Research funding: None declared.

Employment or leadership: None declared.

Honorarium: None declared.
} 
Assessment Questionnaires (SPAQ). Monthly seasonal patterns were analyzed with repeated measures ANOVAs, followed by a post hoc $t$-test if significant. The $\chi^{2}$ was used for presence or absence of seasonal patterns for each item.

Results-More than $75 \%$ of the participants reported at least one seasonal change. More than 75 $\%$ endorsed seasonality in "feeling best" but only $<25 \%$ did so for "feeling worst". Mood-wise, the best month was May, and the worst months were January and February.

Conclusions-There were significant seasonal patterns for all mood and behavior items reported by the majority of participants. The results were consistent with an overall winter pattern of seasonality previously consistently reported in predominantly Caucasian populations.

\section{Keywords}

Old Order Amish; seasonality; Seasonal Pattern Assessment Questionnaires (SPAQ)

\section{Introduction}

Many species on earth experience seasonal changes in physiology and behavior. As a consequence of the tilted axis of rotation of the Earth as it revolves around the Sun, duration of daylight and atmospheric temperature gradually oscillate with a 1 year period. In order for living organisms to survive, they must not only adapt to the environment but also anticipate and prepare themselves for seasonal environmental changes. Over time, humans have partially shielded themselves from seasonal changes in the physical environment by using artificial bright light and controlled micro-climates. Artificial light shortens the duration of exposure to darkness in the winter, artificial heating reduces exposure to cold, and air conditioning in the summer limits exposure to heat. Never theless, some people do experience changes in mood and behavior, although of lesser amplitude than those in many seasonal non-human species. For some humans, these seasonal changes represent a significant problem consisting of seasonal major depressive episodes which are components of a syndrome named seasonal affective disorder (SAD) [1]. The disorder consists of recurrent major depressive episodes during the fall and winter months (termed SAD-winter type) [1] or the spring and summer months (termed SAD-summer type) [2], alternating with spontaneous remissions when the season has ended.

A common instrument used to estimate behavioral seasonality is the Seasonal Pattern Assessment Questionnaire (SPAQ) [3]. Although it is not perfectly suited to diagnose SAD on its own, it is a validated tool that predicts seasonality and screens for SAD diagnosis in individuals [4]. In addition to identifying potential cases of SAD, the SPAQ can also estimate seasonal patterns of mood and behavior in healthy individuals.

The first epidemiological use of the SPAQ was in the form of a telephone survey by Kasper et al. in Montgomery County, Maryland [5]. This group administered the SPAQ to 416 individuals of the population residing in the county. They found that $43.2 \%$ of their participants complained of feeling worst in January and/or February of a given year, and 9.6\% complained of feeling worst in July and/or August. Since then, the SPAQ has been administered across countries, ethnicities, continents and hemispheres [6].

Some healthy individuals may report seasonality that is not severe enough to result in syndromal or subsyndromal SAD diagnosis, but may experience mild seasonality of not only when they feel at their worst, but also when they feel at their best. In addition, healthy individuals may also experience seasonal changes in when they eat the most and when they eat the least, when they gain the most weight and when they lose the most weight, when they 
socialize most and when they socialize least, and when they sleep the most and when they sleep the least.

Individuals with winter type SAD symptoms tend to report eating more, sleeping more, socializing less, and gaining more weight during the winter months [1]. The most prevalent trigger for winter SAD onset is the reduction in day length; this is reversed, at least in part, by exposure to bright light $[1,7,8]$.

The electric light bulb has almost become a necessity of modern living. Until 1809, when Sir Humphrey Davy (1778 - 1829) demonstrated the first carbon arc light, people used light emitted by flames for night-time illumination [9]. Then, in 1878, Thomas Edison (18471931) established the Edison Electric Light Company which was intended to manufacture and operate different devices that would produce light, heat, and power by electricity [9]. With the many products that emerged from this company and others over time, electric power has become an indispensible commodity. Today, there are few cultures that do not use electric light. The one we focus on here is the Old Order Amish.

The lifestyle of the Amish differs greatly from that of their "English " counterparts. The Amish maintain their traditional culture by following the code of the Ordnung, a Pennsylvania-Dutch term for order and discipline, which requires specific conduct for its members. One such prohibited practice is the use of electricity, as they consider it to be an outlet to the outside world, which represents a threat of impingement upon their unique culture. Nonetheless, it is important to note the compromises that the Amish have made in accommodating to modern society. Many have adopted other forms of light sources, such as kerosene lanterns, or, in certain circumstances, battery or generator-based electric devices [10]. There are rare circumstances when Amish people may use electrical light, specifically in instances that require them to interact with non-Amish people. Nevertheless, the Amish are closer to the conditions of our pre-industrial ancestors, being more exposed to the natural variation in day length and temperature changes.

The Amish occupational and social routines may contribute to and even trigger specific seasonal patterns that are unique to them. Farming and agriculture constitutes a major part of the Amish work life; in fact, 50\% of men within 31-50 years engage in farming activities [10]. There are important seasonal patterns of certain social activities in the Amish. For instance, the Amish dedicate a specific time of the year for weddings, which generally occur in November. During this time, there is greater socialization and interaction within the community. This is continued with the winter holidays in December, allowing for increased time for visiting and prayer.

The aim of this study is to analyze the SPAQ monthly patterns of feeling best vs. feeling worst, eating most vs. eating least, gaining the most weight vs. losing the most weight, socializing most vs. socializing least, and sleeping most vs. sleeping least in the Old Order

Amish population of Lancaster, Pennsylvania. This is a component of a larger study, the first SPAQ study in the Old Order Amish, which focuses on estimating prevalence of SAD in the Amish and its genetic epidemiology.

\section{Methods}

Participants of this study were recruited from the Old Order Amish community of Lancaster, Pennsylvania, USA $\left(40^{\circ} 02^{\prime} 23^{\prime \prime}\right.$ North, $76^{\circ} 18^{\prime} 16^{\prime \prime}$ West). The study was approved by the University of Maryland Institutional Review Board (IRB). We contacted the participants via USPS mail by sending a letter inviting them to fill out the SPAQ to the best of their knowledge. The participants received one dollar each for their participation. The 1257 participants of this survey study were part of an original sample of 2254 (response rate of 
$55.8 \%$ ) who participated in several previous cardiovascular health studies and provided consent to be contacted for future research. The responders included 720 females and 537 males, aged 21-99 years old, with an average age of $55(\mathrm{SD}=14)$.

We scored responses to question \#2 on the SPAQ, which asked participants to fill in circles pertaining to all applicable calendar months for the question: "At what time of year do you ..." a) feel best, b) tend to gain most weight, c) socialize most, d) sleep least, e) eat most, f) lose most weight, g) socialize least, h) feel worst, i) eat least, j) sleep most [3].

In order to adjust for multiple month selections on each item, we calculated a ratio representing each item as 1 divided by the number of months the participants reported experiencing it for that particular item. For example, if participants marked one month for the "feel worst" item, 1 point was entered. However, if a participant marked two months for an item, a 0.5 was entered into both months; and if a participant marked four months that he or she "feels worst", a 0.25 was entered for all the pertaining months. Therefore, the participants ' responses on each of the 10 items would equal one full point.

We compared monthly reports for each of the 10 variables (i.e., feel best, feel worst, eat most, eat least, gain most weight, lose most weight, socialize most, socialize least, sleep most, and sleep least) with repeated measures ANOVAs, followed, if signi3 cant, by post hoc t-tests. The $\chi^{2}$ was used for comparing presence vs. absence of monthly pattern.

\section{Results}

\section{Presence vs. absence of any seasonal pattern}

We calculated the number of people who reported any seasonal pattern of mood or behavioral change for each item, as seen in Table 1 . We found that a majority of participants reported a seasonal pattern for when they feel the best; there were $979(77.08 \%)$

participants who reported at least one month of the year in which they feel best. There were a significantly lower number of participants (only 286 or $22.85 \%$ ) who did not report any seasonal pattern for feeling best $\left(\chi^{2}=1187.53, \mathrm{df}=1, \mathrm{p}=0.001\right)$.

In contrast to feeling best, a minority reported seasonality in feeling worst: only 376 (29.72\%) participants reported feeling worst in at least one month out of the year, in contrast to $889(70.28 \%)$ who reported no monthly seasonality in feeling worst $\left(\chi^{2}=208.04, \mathrm{df}=1\right.$, $\mathrm{p}=0.001)$.

There were less than half of the responders who reported a seasonal pattern in months they eat the most; $529(41.82 \%)$ participants reported at least one month in which they eat the most. Slightly more than half of our participants ( 736 or $58.18 \%$ ) did not report any seasonal pattern for eating the most $\left(\chi^{2}=33.87, \mathrm{df}=1, \mathrm{p}=0.001\right)$. There were fewer participants, only $411(32.49 \%)$, who reported at least one month of the year that they eat the least. A majority of participants did not report any monthly seasonal pattern in eating the least; $854(67.51 \%)$ participants did not report any months for which they eat the least $\left(\chi^{2}=155.14, \mathrm{df}=1\right.$, $\mathrm{p}=0.001)$.

There were 591 (46.72\%) participants who reported a monthly seasonal pattern, in which they tend to gain the most weight. Slightly more than half (674 or 53.28\%) of the participants did not report any months in which they gain the most weight $\left(\chi^{2}=5.45, \mathrm{df}=1\right.$, $\mathrm{p}=0.020)$. There were 504 (39.84\%) participants who reported a seasonal pattern, in which they tend to lose the most weight. There were a greater number of participants (761 or $60.16 \%$ ), who did not report any monthly seasonal pattern in which they lose the most weight $\left(\chi^{2}=52.21, \mathrm{df}=1, \mathrm{p}=0.001\right)$. 
The least reported seasonal pattern was for the two items on socialization. Only 402 (31.78\%) participants reported at least 1 month in which they socialize the most. Conversely, there were 863 (68.22\%) participants who did not report any seasonal patterns for socializing the most $\left(\chi^{2}=168.00, \mathrm{df}=1, \mathrm{p}=0.001\right)$. Similarly, there were only 367 (29.01\%) participants who reported at least one month in which they socialize the least, and 898 (70.99\%) who did not report any monthly seasonal pattern for socializing the least $\left(\chi^{2}=222.89, \mathrm{df}=1, \mathrm{p}=0.001\right)$.

Slightly more than half (697 or $55.10 \%$ ) reported at least one month, in which they sleep the most; there were $568(44.90 \%)$ participants that did not report any monthly seasonal patterns for sleeping the most $\left(\chi^{2}=13.15, \mathrm{df}=1, \mathrm{p}=0.001\right)$. There were $761(60.16 \%)$ participants who reported at least 1 month, in which they sleep the least, and 504 (39.84\%) who did not report any monthly seasonal patterns for sleeping the least $\left(\chi^{2}=52.21, \mathrm{df}=1, \mathrm{p}=0.001\right)$.

Overall majority (i.e., 984 or $77.79 \%$ ) of the participants reported at least one change in any of these monthly seasonal patterns, and the minority (i.e., 281 or $22.21 \%$ ) did not report any seasonal patterns $\left(\chi^{2}=390.68, \mathrm{df}=1, \mathrm{p}=0.001\right)$.

\section{Month of the year effect}

Feeling best and feeling worst - There was a significant difference in the months that participants feel best $[\mathrm{F}(11 ; 13,871)=97.87, \mathrm{p}=0.001]$. Participants reported feeling best in May more than any other month $[\mathrm{t}=2.437, \mathrm{p}=0.015]$. There was also a significant difference in months, in which they reported feeling worst $[\mathrm{F}(11 ; 13,871)=36.171, \mathrm{p}=0.001]$.

Participants reported feeling worst in January and Febru-ary more than any other month $[\mathrm{t}=0.191, \mathrm{p}=0.849]$ (see Figure 1A).

Eating most and eating least - There was a significant difference in months, in which the responders reported eating most $[\mathrm{F}(11 ; 13,871)=94.310, \mathrm{p}=0.001]$. They reported eating most in November and December more than any other month, with no difference between them, $[\mathrm{t}=-0.762, \mathrm{p}=0.446]$. There was also a significant difference in the months, in which they reported eating least $[\mathrm{F}(11 ; 13,871)=104.025, \mathrm{p}=0.001]$. Participant reported eating least in July more than any other month, including August [t=3.401, $\mathrm{p}=0.001]$ (see Figure 1B).

Gaining most weight and losing most weight - There was a significant difference in the months, in which the participants reported gaining the most weight $[\mathrm{F}(11 ; 13,871)=462.458$, $\mathrm{p}=0.001]$. They reported gaining the most weight in December more than any other month, including January $[\mathrm{t}=3.054, \mathrm{p}=0.002]$. There was also a significant difference in the months, in which participants reported losing the most weight $[\mathrm{F}(11 ; 13,781)=89.655, \mathrm{p}=0.001]$. They reported losing the most weight in July $[\mathrm{t}=2.645, \mathrm{p}=0.008]$ more than any other month (Figure 1C).

Socializing most and socializing least - There was a significant difference in the months that responders reported socializing most $[\mathrm{F}(11 ; 13,871)=43.605, \mathrm{p}=0.001]$. Participants reported socializing most in November [ $\mathrm{t}=5.388, \mathrm{p}=0.001]$. There was a significant difference in months, in which the responders reported socializing least $[\mathrm{F}(11 ; 13,871)=75.647, \mathrm{p}=0.001]$. They reported socializing least in January and February more than any other month, with no difference between them $[t=1.415 \mathrm{p}=0.175]$ (see Figure 1D).

Sleeping most and sleeping least - There was a significant difference in months, in which the participants reported sleeping most $[\mathrm{F}(11 ; 13,871)=482.252, \mathrm{p}=0.001]$. They reported sleeping most in January $[\mathrm{t}=10.893, \mathrm{p}=0.001]$ more than any other month. There was also a significant difference in months, in which they reported sleeping least

$[F(11 ; 13,871)=206.968, p=0.001]$. They reported sleeping least in June and July more than 
any other month, with no difference between the two peak months $[\mathrm{t}=0.740, \mathrm{p}=0.460]$ (see Figure 1D).

\section{Discussion}

Majority (>75\%) of the Amish participants reported some form of seasonal change in mood and behavior, and only a minority reported no seasonal change $(<25 \%)$, as shown in Table 1 . The percentage of Amish participants who reported at least one month for a noticeable seasonal pattern was highest for "feel best". In other words, while $77 \%$ of the participants reported seasonality of "feeling best ", $70 \%$ did not report any seasonality in " feeling worst". This might be attributed to certain Amish values of thankfulness and modesty, focusing on the positive rather than the negative, and a negative value attached to complaining. The Amish word Gelassenheit, which most nearly translates as submission to a higher power - whether it is community, religion, or tradition - reflects a focus on the community rather than on the individual [10]. An acknowledgment of feeling worst may appear ungrateful or reveal a preoccupation with one's self. The Amish value stability, a willing submission to their tradition, and the attribution that "stability is credited to their strong positive interest" [11] in their society. Many Amish individuals may perceive positively answering the question "When do you feel worst? " as reflecting an individual dissatisfaction with life that goes against their ideal of humility and communal identification. In comparison, the recognition of months during which one feels best connotes thankfulness and contentment.

In our study of SAD in the Amish, $0.84 \%$ of the Amish met SPAQ criteria for winter SAD and $2.59 \%$ met criteria for total SAD (defined as the presence of either winter SAD or a milder form called subsyndromal SAD, or s-SAD) [12]. One of the possible explanations for the low prevalence of SAD in the Amish involves the cultural factors discussed above, particularly gelassenheit and the denial of dissatisfaction with life in response to the question about seasonality pattern as determined by the months during which the participant felt worst. To take this into account, we reviewed the responses for participants who met the GSS and problem criteria for SAD but who did not indicate the season in which they felt worst, preferring only to indicate the months in which they felt best. We made the assumption that this might be a problem in reporting based on cultural factors where participants did not wish to admit that there were times during which they felt "worst". We made a further assumption that the months in which these participants felt worst would be during the time where the season was opposite to the season in which they felt best, i.e., by taking the month six months prior to or after the month in which they reported to feel best. By doing this, we found an additional three cases who met the SPAQ criteria for SAD and 10 cases who met the SPAQ criteria for s-SAD. Despite this speculative adjustment, which increased the prevalence to $1.1 \%$ for SAD and $3.6 \%$ for total SAD, the prevalence of SAD and total SAD in the Amish remained below that of most other SPAQ based studies. It is also possible that there were individuals who met the GSS and seasonal pattern criteria of SAD but who did not meet the problem criterion, although we did not account for such cases. We also did not consider any other seasonal changes except the months during which participants felt worst. In that study, we only analyzed cases who met the criteria for SAD or s-SAD, whereas this study examines seasonal changes in mood, sleep length, socialization, weight, and appetite in all participants, healthy or otherwise. The only parameter of seasonality not assessed in this study is seasonal changes in energy level of the participants.

We found significant seasonality patterns for all 10 categories of change. We found that, on average, responders tend to feel best during the end of spring and just before summer, while they tend to feel worst during winter. It is important to mention that, although the day length in November and December is generally shorter than in January and February, the rate of 
feeling worst was not as elevated in November and December, potentially as a result of increased socializing during the wedding season (November) and winter holidays (December). It must be noted, though, that non-Amish participants in Montgomery County, Maryland, also felt worst in January and/or February [5], although the percentage of those feeling worst in these months was higher (43\%) compared to the Amish (slightly over 10\%). In addition to January and February, a smaller peak in "feeling worst" occurs in July and August (see Figure 1A), possibly as a result of exposure to heat increased in the Amish occupationally and not opposed by the use of air conditioning. There is a comparable peak in Montgomery County in July/August [5] although the magnitude is smaller in the Amish (10\% in Montgomery County vs. about $7.5 \%$ in the Amish). Also notable is the fact that the ratio of those who feel worst in winter to those who feel worst in summer is much larger in Montgomery County (4.5:1) than in the Amish (approx. 1.5:1). What role non-exposure to air conditioning may play in the Amish is unclear. During the summer season, there was a greater report of eating least, losing most weight, and sleeping least. Furthermore, the opposite behaviors, i.e., sleeping most, eating most, gaining most weight, and socializing least were distributed as mirror image to sleeping least, eating most, and losing weight.

Given that they are less shielded from exposure to natural light and seasonal changes in temperature than the majority of people in the developed country, it is important physiologically to study seasonality of mood and behavior in the Old Order Amish.

A strong point of this study is that it proves the answers to the mood and behavior questions have face validity, as there is a purposeful mirror image of monthly season patterns in antonym items (Figure 1). The peaks in one behavior approximately correspond to troughs in the opposite behavior, and vice-versa.

It is important to note that this study analyzes more than just the "worst month", as reported by other studies focused on SAD symptoms [13-17], but includes other characteristics of seasonality. Most of all, this study analyzed the seasonality of "feeling best", which has been neglected in previous SPAQ studies, even though it is relevant in a population more inclined to focus on the positive.

Limitations to this study begin with the nature of the SPAQ. This is a paper and pencil type of self-report questionnaire. This type of questionnaire requires individual recall of seasonal patterns, which may be less than adequate [5]. The response rate was limited in this study. Many other studies have found lower response rates when mailing the SPAQ to participants, than when conducting either a phone or face-to-face interview [16 - 18]. Nevertheless, because response rates in SPAQ-based studies have ranged from about $14 \%$ to $90 \%$, with an average response rate of approximately 50\% [5], the response rate of our study has been comparable to most SPAQ-based studies. It is also possible that our sample may not be representative of the full population, and that more people who have a seasonal variation in symptoms may have been more inclined to respond than non-symptomatic people. Further, this instrument has not yet been validated for use in this population. Nevertheless, because this is the first study to administer a seasonality questionnaire in the Amish, we believe that its contributions to knowledge outweigh its limitations.

The major strengths of this report, are the reliability of the Amish in answering the seasonality questions (as illustrated by the internal validity suggested by the mirror images illustrated in Figure 1), and the limited use of electric light and air conditioning by the Amish, thereby reflecting reduced isolation from the seasonal changes of the natural environment in the studied population. In addition, as the Old Order Amish are prohibited from consuming alcohol, the component of seasonality potentially resulting from the 
seasonal variation in alcohol use [19] is likely to be less relevant in the current study than in previous studies in the general population.

In conclusion, a majority of the Amish report at least some seasonal variations in mood and behavior. The monthly patterns of mood, socializing, eating, and sleeping emerged in the Old Order Amish characterized by a predominance of positive feelings, eating and sleeping less, and socializing more during summer months, as well as a predominance of negative feelings, eating and sleeping more, and socializing less during winter months; these confirm a predominantly winter type of seasonal patterns as previously reported in predominantly Caucasian populations [6].

\section{Acknowledgments}

The study was supported by an NIH grant K18MH093940 (PI Postolache). The authors acknowledge Drs. Alan Shuldiner and Braxton Mitchell for their guidance and mentorship in Amish research; Drs. Aamar Sleemi and Gagan Virk Nijjar as well as Hassaan Yousufi for their help in mailing out the questionnaires, in IRB-related issues, and in database management. We also thank Francis Iyoriobhe, Christine Ballwanz, Kathleen Ryan, as well as the staff of the University of Maryland Amish Research Clinic (Lancaster, PA) for their essential support for the study.

\section{References}

1. Rosenthal NE, Sack DA, Gillin C, Lewy AJ, Goodwin FK, Davenport Y, et al. Seasonal affective disorder, a description of the syndrome and preliminary findings with light therapy. Arch Gen Psychiatry. 1984; 41:72-80. [PubMed: 6581756]

2. Wehr TA, Sack DA, Rosenthal NE. Seasonal affective disorder with summer depression and winter hypomania. Am J Psychiatry. 1987; 144:1602-3. [PubMed: 3688288]

3. Rosenthal, NE.; Genhart, MJ.; Sack, DA.; Skwerer, RJ.; Wehr, TA. Seasonal affective disorder and its relevance for the understanding and treatment of bulimia. In: Hudson, JI.; Pope, HG., Jr, editors. The psychobiology of bulimia. Washington, DC: American Psychiatric Press; 1987. p. 205-28.

4. Thompson C, Stinson D, Fernandez M, Fine J, Isaacs G. A comparison of normal, bipolar and seasonal affective disorder subjects using the Seasonal Pattern Assessment Questionnaire. J Affect Disord. 1988; 14:257-64. [PubMed: 2968387]

5. Kasper S, Wehr TA, Bartko JJ, Gaist PA, Rosenthal NE. Epidemiological findings of seasonal changes in mood and behavior: a telephone survey of Montgomery County, Maryland. Arch Gen Psychiatry. 1989; 46:823-33. [PubMed: 2789026]

6. Magnusson A. An overview of epidemiological studies on seasonal affective disorder. Acta Psychiatr Scand. 2000; 101:176-84. [PubMed: 10721866]

7. Terman M, Terman JS, Quitkin FM, McGrath PJ, Stewart JW, Rafferty B. Light therapy for seasonal affective disorder: a review of efficacy. Neuropsychopharmacol. 1989; 2:1-22.

8. Eastman CI, Young MA, Fogg LF, Liu L, Meaden PM. Bright light treatment of winter depression: a placebo-controlled trial. Arch Gen Psychiatry. 1998; 55:883-9. [PubMed: 9783558]

9. Williams, B. A history of light and lighting. Rutgers University; 1990. URL: http://www.mts.net/ $\sim$ william5/history/hol.htm

10. Kraybill, DB. The riddle of Amish culture. Baltimore: The Johns Hopkins University Press; 2001.

11. Ediger M. Teaching about another culture: the Old Order Amish. Social Studies. 1996; 87:161.

12. Raheja UK, Stephens SH, Mitchell BD, Rohan KJ, Vaswani D, Balis TG, et al. Seasonality of mood and behavior in the Old Order Amish. J Affect Disord. 2012 Nov 16. pii: S0165-0327(12)00698-2. [Epub ahead of print]. 10.1016/j.jad.2012.10.019

13. Murray G. The seasonal pattern assessment questionnaire as a measure of mood seasonality: a prospective validation study. Psychiatry Res. 2003; 120:53-9. [PubMed: 14500114]

14. Mersch PP, Middendorp HM, Bouhuys AL, Beersma DG, van der Hoofdakker RH. The prevalence of seasonal affective disorder in the Netherlands: a prospective and retrospective study of seasonal mood variation in the general population. Biol Psychiatry. 1998; 45:1013-22. [PubMed: 10386184] 
15. Hedge AL, Woodson H. Prevalence of seasonal changes in mood and behavior during the winter months in central Texas. Psychiatry Res. 1996; 62:265-71. [PubMed: 8804137]

16. Muscettola G, Barbato G, Ficca G, Beatrice M, Puca M, Uguglia E, et al. Seasonality of mood in Italy: role of latitude and sociocultural factors. J Affect Disord. 1995; 33:135-9. [PubMed: 7759662]

17. Rosen L, Targum SD, Terman M, Bryant MJ, Hoffmann H, Kasper S, et al. Prevalence of seasonal affective disorders at four latitudes. Psychiatry Res. 1990; 31:131-44. [PubMed: 2326393]

18. Morrisey SA, Raggatt PT, James B, Rogers J. Seasonal affective disorder: some epidemiological findings from a tropical climate. Aust NZ J Psychiatry. 1996; 30:579-86.

19. Sher L. Alcoholism and seasonal affective disorder. Compr Psychiatry. 2004; 45:51-6. [PubMed: 14671737] 



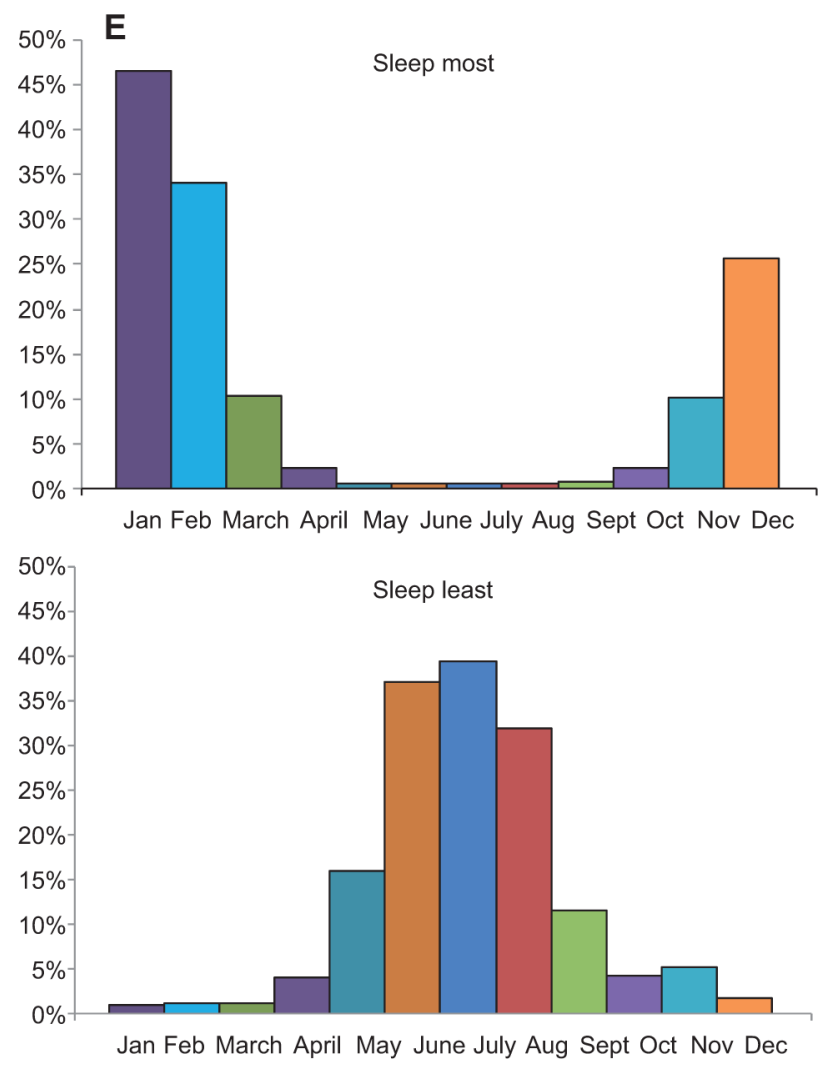

Figure 1.

Monthly season patterns in antonym items. (A) Monthly variation in feeling best and feeling worst ( $\%$ of individuals). (B) Monthly variation in eating most and eating least ( $\%$ of individuals). (C) Monthly variation in gaining most weight and losing most weight (\% of individuals). (D) Monthly variation in socializing most and socializing least (\% of individuals). (E) Monthly variation in sleeping most and sleeping least (\% of individuals). 
